\title{
Rotator Cuff Tendinosis in an Animal Model: Role of Extrinsic and Overuse Factors
}

\author{
Louis J. Soslowsky, ${ }^{1}$ Stavros Thomopoulos, ${ }^{1}$ Adil Esmail, ${ }^{1}$ ColleEn L. Flanagan, ${ }^{1}$ \\ JOSEPH P. IANNOTTI, ${ }^{1}$ J. DAVID WILLIAMSON III, ${ }^{1}$ and JAMES E. CARPENTER ${ }^{2}$ \\ ${ }^{1}$ McKay Orthopaedic Research Laboratory, University of Pennsylvania, Philadelphia, PA and ${ }^{2}$ Orthopaedic Research Laboratories, \\ University of Michigan, Ann Arbor, MI
}

(Received 15 February 2002; accepted 22 July 2002)

\begin{abstract}
The rat shoulder animal model has been used previously to study the role of intrinsic injury (modeled as an acute insult to the tendon), extrinsic injury (modeled as external subacromial impingement), and overuse factors on rotator cuff tendinosis. These studies demonstrated that it is possible to produce rotator cuff tendinosis with any one of these factors in isolation. The current study uses the rat shoulder model to study the roles of extrinsic compression, overuse, and overuse in combination with extrinsic compression, on the development of rotator cuff tendinosis. The results of this study demonstrate that the injury created by overuse plus extrinsic compression is greater than the injuries created by overuse or extrinsic compression alone, particularly when important biomechanical variables are considered. While ineffective in causing a change in supraspinatus tendon properties in animals with normal cage activity, extrinsic compression had a significant and dramatic effect when it was combined with overuse activity. Without an additional factor, such as overhead activity, the extrinsic compression alone may be insufficient to cause tendinosis. The results of the present study support the role of multiple factors in the etiology of some rotator cuff injuries. (C) 2002 Biomedical Engineering Society. [DOI: 10.1114/1.1509765]
\end{abstract}

Keywords-Shoulder, Mechanical properties, Tendon, Supraspinatus, Tendonitis, Tendonosis, Rotator cuff, Impingement, Overuse.

\section{INTRODUCTION}

The rotator cuff tendons of the shoulder are among the most commonly injured musculoskeletal soft tissues. Occupational injury to the shoulder ranks second only to lower back pain in clinical frequency. ${ }^{2}$ Despite the magnitude and economic impact of rotator cuff injuries, little is known concerning their etiology. Previous studies examining rotator cuff injuries have focused on the surgical or clinical retrospective perspective. ${ }^{5,12,13,20,21}$ These

Address correspondence to Louis J. Soslowsky, PhD, McKay Orthopaedic Research Laboratory, University of Pennsylvania, 424 Stemmler Hall, Philadelphia, PA 19104-6081. Electronic mail: soslowsk@mail.med.upenn.edu studies, while valuable, cannot test specific hypotheses regarding the etiology or pathogenesis of rotator cuff tendinosis.

Previous rotator cuff studies can be categorized by whether they support intrinsic or extrinsic mechanisms of injury. ${ }^{1,5,8,10,11,14}$ The intrinsic mechanism has been defined as a tendon injury that originates within the tendon from direct tendon overload, intrinsic degeneration, or other insult. The extrinsic mechanism has been defined as tendon damage caused by injury of the tendon through compression against surrounding structures, most specifically, the coracoacromial arch. Overuse activity has also been implicated as an important etiologic factor (note that some investigators categorize overuse as one type of intrinsic injury). Each of these individual factors cannot explain or predict the full range of injuries seen clinically. Patients may have one factor present (e.g., a bony anatomy indicative of extrinsic compression or a history of overuse activities) and not develop tendinosis. A combination of factors may be more likely to result in tendinosis. These proposed factors, however, have not been addressed explicitly in any controlled experimental studies. Different approaches toward prevention and treatment of rotator cuff disease might be appropriate depending on which mechanism is dominant in a particular injury.

Our previous study has identified the rat as an appropriate in vivo animal model in which to study rotator cuff disease. ${ }^{18}$ This model is particularly suited to such studies due to several anatomic characteristics. Most importantly, the rat shoulder has an enclosed arch through which the supraspinatus tendon must pass, comparable to the coracoacromial arch found in the human. This animal model has been used previously to study the role of intrinsic injury (modeled as an acute insult to the tendon), ${ }^{3,18}$ extrinsic injury (modeled as external subacromial impingement), ${ }^{3,18}$ and overuse factors ${ }^{19}$ on rotator cuff tendinosis. These studies demonstrated that it is possible to produce rotator cuff tendinosis via isolated in- 
trinsic, extrinsic, or overuse injury. Previous investigations using this animal model, however, were not designed to compare the effect of combinations of factors on the development of tendinosis. The appropriate single factor controls were not available for appropriate statistical comparison of the effect of each factor alone or in combination. The multifactorial nature of rotator cuff pathology is the focus of the current study. The rat shoulder model is used in this study to examine the roles of extrinsic compression, overuse, and overuse in combination with extrinsic compression, on the development of rotator cuff tendinosis. It was hypothesized that a single injury factor would be insufficient to create persistent tendinosis and that a combination of injury factors would be necessary. This hypothesis was based on the clinical presentation of shoulder pathology, and was not necessarily based on our previous work.

\section{MATERIALS AND METHODS}

108 mature (but not aged) Sprague-Dawley rats $(530 \pm 43 \mathrm{~g})$ were divided evenly into three groups ( $n$ $=36$ each) and animals either received extrinsic compression $(\mathrm{E})$, overuse $(\mathrm{OV})$, or a combination of both overuse and extrinsic compression (OV/E).

To model extrinsic compression (E and OV/E groups), the left supraspinatus tendons were surgically exposed and an Achilles tendon allograft was wrapped around the acromion, such that it lay directly above the supraspinatus tendon to provide extrinsic compression. This procedure has been used successfully in previous studies ${ }^{3}$ to create an injury and will be briefly summarized below. Rats were anesthetized via inhalation of isofluorane gas. All surgery was performed using standard aseptic operating room protocols. To alter the acromion and to simulate an impingement lesion, an Achilles tendon allograft was utilized. These allograft tendons were harvested from donor rats. The donor tendons were aseptically removed, immediately frozen in liquid nitrogen, and stored at $-80^{\circ} \mathrm{C}$. This treatment was effective in preventing any immune response from the receiving animal based on gross and histologic evaluation. Surgical exposure of the supraspinatus tendon was achieved as follows. A 2 $\mathrm{cm}$ skin incision was made over the craniolateral aspect of the scapulohumeral joint. Sharp dissection of the deltotrapezius fascia was performed to expose the supraspinatus tendon. The supraspinatus tendon was visualized as it passed through the bony arch created by the acromion, coracoid, and clavicle to its insertion on the greater tuberosity of the proximal humerus. In the left shoulder, a thawed tendon allograft was inserted so that it lay just underneath the acromion between the acromion and supraspinatus tendon. The allograft was then wrapped over the superior surface of the acromion and sutured, thus forming a loop. The two free ends of the allograft were sutured together (4-0 suture, simple interrupted pattern). The overlying deltoid muscle was then sutured back to the trapezius muscle with 4-0 sutures. In the right shoulder a sham procedure was performed. The acromion was exposed in an identical fashion as the contralateral side. A pair of hemostats was then gently inserted proximally to distally underneath the bony arch, passing ventrally to the acromion, to simulate passing of the tendon graft. Again, the deltoid and trapezius muscles were repaired. Closure of the surgical sites was made in a routine manner. The muscle and fascia was closed with 4-0 Vicryl suture using a simple interrupted pattern. The skin was closed with surgical staples. Postoperatively, animals were transferred to a recovery cage and monitored for adverse signs. Staples were removed 7-10 days postsurgery.

To model overuse (OV and OV/E groups), rats were trained to run downhill on a custom treadmill at 17 $\mathrm{m} / \mathrm{min}$ for $1 \mathrm{~h} /$ day, 5 days/week as previously described. ${ }^{16,19,22}$ For rats in the combination group (OV/ E), a 1 week postoperative recovery period was allowed prior to the overuse protocol. Following this recovery period, rats were returned to full speed overuse activity over the course of 2 weeks.

Rats in each group were sacrificed at either 4 weeks $(n=12), 8$ weeks $(n=12)$, or 16 weeks $(n=12)$. An additional 10 rats were allowed normal cage activity (CA) and served as an uninjured control group.

For histologic analysis, two rats from each time point were randomly selected. Two animals were deemed sufficient for this qualitative histologic assessment. The supraspinatus tendons from both the right and left forelimbs were fixed, processed, embedded, sectioned, and stained using hematoxylin and eosin. Sections were then graded for cellularity, cell shape, and collagen fiber organization as described previously. 3,4,18,19 This analysis was accomplished in a semiquantitative way by assigning a rank to each of the observations where 0 indicates normal, 1 indicates mild changes, 2 indicates moderate changes, and 3 indicates marked changes. The histologic analysis was performed independently by three graders, masked to specimen group, on representative sections from each of these tendons. For each variable examined, measures from the three graders were averaged. An overall histologic grade for the tissue sections was also obtained from a combined average of the grades for each variable.

For geometrical and biomechanical analysis, ten rats from each time point were randomly selected and fresh frozen supraspinatus tendons from both the right and left forelimbs were excised. A power analysis was performed on preliminary data to determine the appropriate number of specimens to use for this quantitative assay. Tendon thickness was quantified using a device consisting of an indenter probe attached to a high-resolution linear vari- 
able differential transformer. ${ }^{3,4,18,19}$ Tendon width was obtained using an optically based image processing system. ${ }^{3,4,18,19}$ Previous studies indicated that tendons are flat and wide. Cross-sectional area was, therefore, calculated as the product of average specimen width and average specimen thickness (the large aspect ratio of thickness to width makes any overestimation due to rounded edges minimal when assuming a rectangular crosssectional area). For biomechanical testing, the area of interest in the supraspinatus tendon was prepared as a standardized dumbbell-shaped specimen $(3.5 \mathrm{~mm}$ gage length) using well-established techniques. The dumbbellshaped specimens were made using a razor blade stamp. This geometry was created to ensure failure of the specimens in the tendon midsubstance. The cross-sectional area was measured again after stamping for use in calculation of stress. To avoid any complications regarding the myotendinous junction, all of the supraspinatus muscle fibers were gently teased off of their tendon attachment. The humerus was embedded in PMMA in custom designed fixtures and the proximal end of the tendon was held in a spring-loaded clamp lined with fine grit sandpaper. Because of the small thickness, we are confident that all fibers through the depth of the tendon were loaded consistently. A stain line was placed on the tendon just inside the grip, to track the possibility of tendon slipping out of the grip. Slipping was not noted in any specimen. The specimen was tested to failure in uniaxial tension using a custom built testing system at a constant elongation rate of $14 \mu \mathrm{m} / \mathrm{s}(\sim 0.4 \% / \mathrm{s})$ in a $39^{\circ} \mathrm{C}$ PBS bath (rat body temperature). Supraspinatus tendons tested in previous studies displayed typical mechanical behavior for biologic tissues. There was an initial nonlinear "toe region" at low loads, followed by a "near-linear region" at intermediate loads, and followed by a "failure region" at higher loads. The tissue modulus was calculated by performing a linear regression on the near-linear region of the stress strain curve (preliminary studies indicated $r^{2}$ values for this region consistently above 0.9 ). The maximum stress was calculated from the maximum load achieved in the test.

Geometric and mechanical data were statistically analyzed. Statistical comparisons were made within group to determine the effect of injury factor compared to control and between groups to compare the effect of single injury factors to multiple injury factors. Comparisons within the E group were made between left (surgically injured) tendons and right (sham operated) tendons. Comparisons within the $\mathrm{OV}$ and $\mathrm{OV} / \mathrm{E}$ groups were made with uninjured, cage activity control tendons since no contralateral sham exists. To compare the effect of single injury factor groups $(\mathrm{OV}, \mathrm{E})$ to the multiple injury factor group $(\mathrm{OV} / \mathrm{E})$, it was necessary to take into account the appropriate control data for each group and normalize the data. Data for E were normalized by con- tralateral control data for each animal. Data for OV and OV/E were normalized using a group of cage activity control animals (CTL) because no contralateral shoulder is available. Animals from this control group were paired with animals from the experimental groups ( $\mathrm{OV}$ and $\mathrm{OV} / \mathrm{E}$ ) using a bootstrapping technique to provide the most rigorous statistical comparison of our data. Bootstrapping is a standard statistical technique that allows for the normalization of one data set with an unpaired second data set. In the case of the E group, bootstrapping is unnecessary, as each data point (e.g., the modulus result from rat No. 377, left supraspinatus tendon) can be normalized by the paired data from the contralateral limb (e.g., the modulus result from rat No. 377, right supraspinatus tendon). For the other two groups (OV and OV/E), however, a "paired data set" does not exist. It is inappropriate to simply take the average results from one group (OV or OV/E) and normalize with the average results from the second group (CTL). Therefore, a worksheet was created to form 11 random pairings between the injury groups $(\mathrm{OV}$ and $\mathrm{OV} / \mathrm{E})$ and the normalization group (CTL). Average and standard deviation was then calculated for each of the 11 sets of normalized results. The median result was then used for comparison. Normalized data from single injury factor groups (E, OV) were compared to normalized data from the multiple injury factor group $(\mathrm{OV} / \mathrm{E})$ using Student t-tests. The $\alpha$ significance level was set to $p<0.05$.

\section{RESULTS}

The histologic grades of the three investigators agreed extremely well (indicating repeatability in the grading system). Histologic evaluation of the tissue revealed slight changes in the $\mathrm{E}, \mathrm{OV},{ }^{19}$ and $\mathrm{OV} / \mathrm{E}$ group relative to normal (Fig. 1, Table 1). There were no apparent changes over time in any group. Histologic changes were consistent with those seen clinically in human rotator cuff tendinosis (increased cellularity, rounder cell shape, and decreased collagen organization). The actual numerical grades were purposefully omitted to accentuate the qualitative nature of the histology results. Note that all OV data were taken from a previously published study. ${ }^{19}$

The effect of each injury model (OV, E, and OV/E) was statistically compared for each quantitative variable assessed (cross-sectional area, maximum stress, and modulus) at each time point studied $(4,8$, and 16 weeks). Note that the results for OV were taken from Ref. 19. For the E group, the injured shoulder was compared with its contralateral control. This comparison resulted in no significant differences (at $p<0.05$ ), demonstrating that the injury model "E alone" did not produce a consistent injury based on these quantitative variables (Fig. 2). For the OV and OV/E groups, the injured shoulders were compared with their appropriate control group 


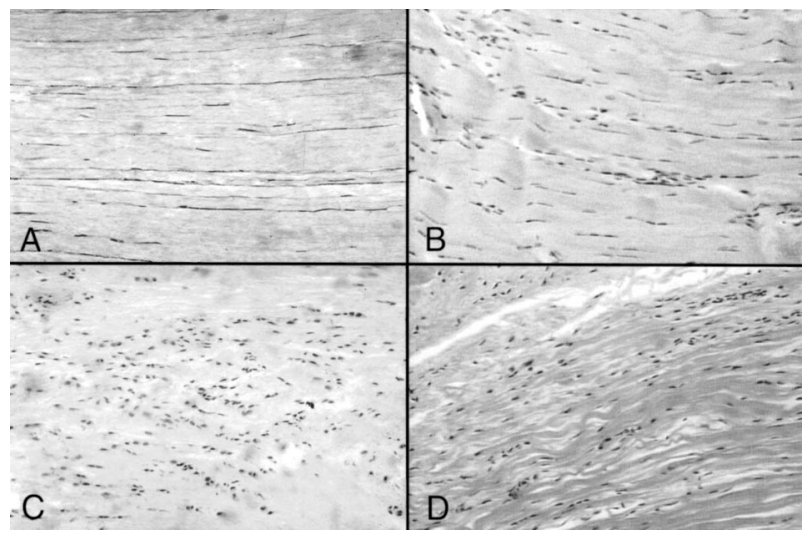

FIGURE 1. Histology from the 8 week time point for (A) uninjured control (CTL), (B) extrinsic alone (E), (C) overuse alone (OV), and (D) extrinsic combined with overuse (OV/E). All injury groups (E, OV, and OV/E) demonstrated apparent differences compared to uninjured control (CTL), consistent with changes seen clinically in human rotator cuff tendinosis (hemotoxylin and eosin stain, 20X objective).

(CTL). For both of these groups, a statistically significant increase $(p<0.05)$ was present in cross-sectional area as well as a statistically significant decrease $(p<0.05)$ in maximum stress and tissue modulus at all time points (Fig. 2). These results indicate that OV and OV/E injury factors produced consistent injuries at each of the time points studied.

At 4 weeks, OV/E demonstrated a significant increase in tendon cross-sectional area compared with $\mathrm{E}$ or OV alone and a significant decrease in tissue maximum stress and modulus compared with $\mathrm{E}$ or OV alone (Fig. 2). At 8 weeks, OV/E demonstrated a significant increase in area compared with $\mathrm{E}$ or $\mathrm{OV}$ alone and a significant decrease in maximum stress and modulus compared with E or OV alone (Fig. 2). At 16 weeks, OV/E demonstrated a significant increase in area compared to $\mathrm{E}$ or $\mathrm{OV}$ alone, and a significant decrease in maximum stress and modulus compared to $\mathrm{E}$ or $\mathrm{OV}$ alone (Fig. 2).

There were no significant changes over time in the $\mathrm{E}$ or OV/E groups in any variable. There were no significant changes over time in maximum stress or modulus in

TABLE 1. Summary of histologic, geometric, and biomechanical changes. Changes noted are relative to the appropriate controls.

\begin{tabular}{lcccc}
\hline Histology & $\begin{array}{c}\text { Cross-sectional } \\
\text { area }\end{array}$ & $\begin{array}{c}\text { Max. } \\
\text { stress }\end{array}$ & $\begin{array}{c}\text { Tissue } \\
\text { modulus }\end{array}$ \\
\hline $\mathrm{E}$ & $\begin{array}{c}\text { slight } \\
\text { changes } \\
\text { OV }\end{array}$ & $\leftrightarrow$ & $\leftrightarrow$ & $\leftrightarrow$ \\
OV/E & $\begin{array}{c}\text { changes } \\
\text { slight } \\
\text { changes }\end{array}$ & $\uparrow \uparrow$ & $\downarrow$ & $\downarrow$ \\
\hline
\end{tabular}
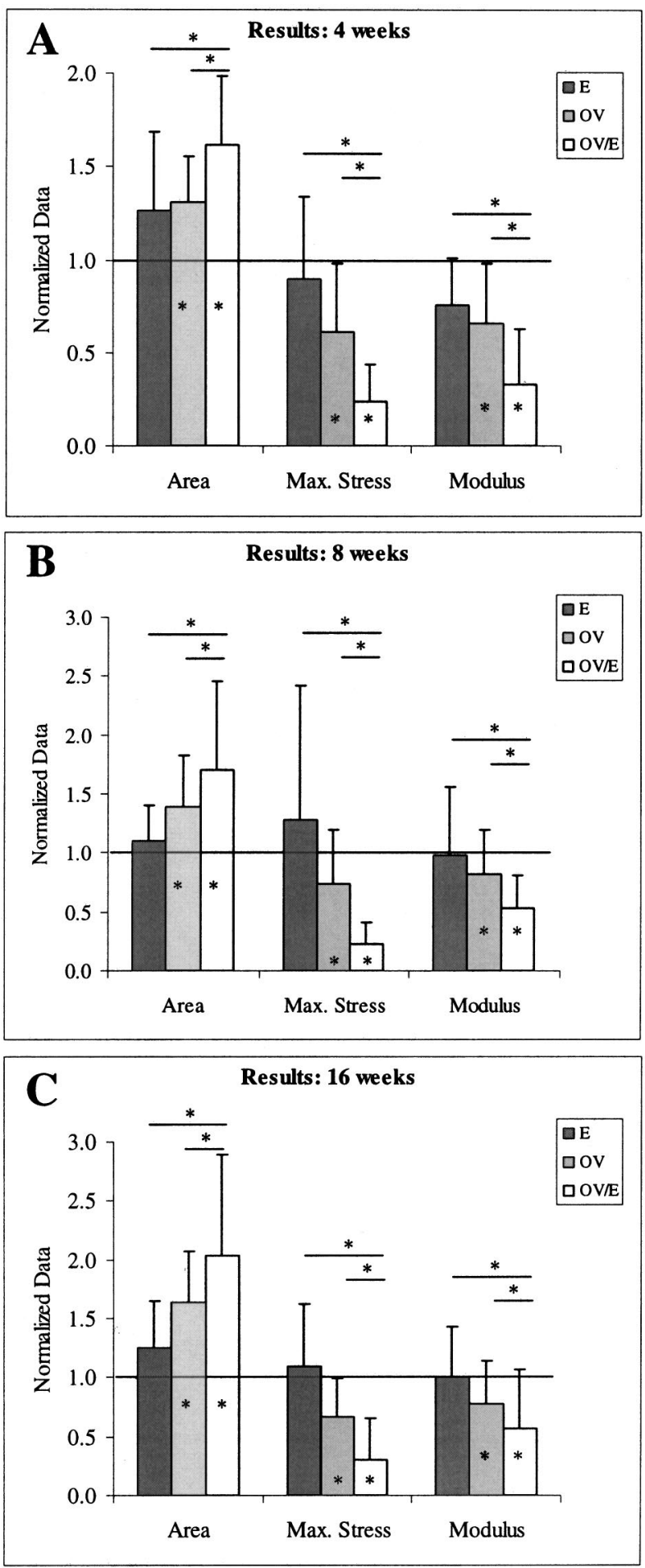

FIGURE 2. Injury created by overuse plus extrinsic compression is greater than the injuries created by overuse or extrinsic compression alone. Significant differences relative to control are indicated by a "*" inside each bar (average \pm standard deviation). Significant differences between single factors $(E, O V)$ and multiple factors (OV/E) are indicated by a "*" above each pair of bars. 
the OV group. There was a statistically significant increase in area between 4 and 16 weeks in the OV alone group. ${ }^{19}$

\section{DISCUSSION}

In the current study, an animal model was used to address the role of single factors as well as combinations of factors on rotator cuff tendinosis. Previous studies have supported either intrinsic, ${ }^{3,5,8,10,11,14,20,21}$ extrinsic, $^{1,3,5,13,23}$ or overuse factors ${ }^{3,6,7,9,15,16,19}$ for tendon injury. Our study examined the effect of overuse activity and extrinsic compression on the supraspinatus tendons of rats.

The results of this study demonstrate that the injury created by overuse plus extrinsic compression is greater than the injuries created by overuse or extrinsic compression alone, particularly when the important biomechanical variables of maximum stress and modulus are considered. While ineffective in causing a change in properties in animals with normal cage activity, extrinsic compression had a significant and dramatic effect when it was combined with overuse activity. In other words, an anatomy that causes extrinsic compression did not cause injury until overuse activity was introduced. This result has important clinical relevance with regards to the influence of the extrinsic compression and overuse injury factors. In the clinical setting, a bony anatomy indicating the presence of extrinsic compression, such as a hooked or a type III acromion, ${ }^{1}$ may not necessarily cause the patient to develop rotator cuff tendinosis. Without an additional factor, such as overhead activity, the extrinsic compression alone may be insufficient to cause tendinosis. However, these results suggest that extrinsic compression would predispose an individual to tendinosis if combined with overuse activities resulting in a greater degree of tendon injury than either compression from the coracoacromial arch or overuse alone. The results of the present study support the role of multiple factors (e.g., overuse coupled with extrinsic compression) in the etiology of some rotator cuff injuries.

In our model, there were few improvements over time in any of the injury groups. This is indicative of a persistent tendinosis condition. The injury was incited within the first four weeks after the injury factor was introduced, and remained throughout the period of study (16 weeks). The tissue is, therefore, unable to mount a successful response to the factors introduced. Future studies will attempt to treat the tendinosis condition by removing the injury factors. We hypothesize that with the tendinosis factors removed, either through rest (for the overuse factor) or through acromioplasty (for the extrinsic compression factor), the properties of the tissue will return to normal over time.
To test the hypothesis proposed for this study (compare the effect of single injury factors to multiple injury factors) appropriate control data for each group were used to normalize the data for that group, an approach which differed from that in our previous study examining the etiology of rotator cuff tendinosis. ${ }^{3}$ Additionally, the comparison groups used in this study differed slightly from those used in our previous study, which also compared multiple injury factors. ${ }^{3}$ The previous study used unoperated contralateral shoulders to represent an "overuse only" group. It can be argued, however, that animals may have used this unoperated shoulder preferentially during treadmill activity, making its use as a control questionable. The current study contained a group of overuse alone animals (without any surgical manipulation) for comparison to the combination group, a sham operated contralateral limb for use as a control and for normalization for the extrinsic alone group, and a cage activity group (without surgical manipulation) for use as a control and for normalization for the overuse alone group, in order to address the limitations of the previous study design. These important differences make the current study distinct from previous work and provide a more rigorous statistical test of the proposed hypothesis.

There were a variety of options to consider when choosing which biomechanical measurements to make in this study, including: tensile versus compressive properties, elastic versus viscoelastic properties, and structural versus material properties. The variables modulus and failure stress were chosen in order to remain consistent with the literature and to present a straightforward yet appropriate assessment of the tissue. While compressive properties may be important, tensile properties are more relevant properties in a tissue such as tendon, whose primary function is to transfer muscle forces along one direction. While knowledge of the compressive properties of the region below the extrinsic impingement would be useful, the first assessment of this tissue should be in the tensile direction, as that is believed to be the predominant loading direction in this tissue. Elastic properties derived from a constant strain rate test to failure were, therefore, chosen to stay consistent with the large body of literature available for the mechanical properties of tendons and ligaments. Viscoelastic properties will be evaluated in future studies. Finally, true structural properties were not measured as a result of our methodology, which involved changing the geometry of the tissue (recall that tendons were stamped into dumbbell-shaped specimens). Pilot studies indicated that intact specimens did not fail in the tendon midsubstance, but failed either at the grip or at the humeral head growth plate (rat growth plates do not fuse). A choice was made to forego knowledge of the structural properties stiffness and maximum load, to be able to measure the material properties modulus and failure stress. It was decided that 
failure properties were important enough to forego knowledge of structural properties. For information on a condition such as tendinosis, material properties are more important to consider, as they indicate the condition of the tissue, and not the overall structure. These are relevant variables because a change in material that produces an inferior tissue (as indicated by decreased properties), indicates a disease state. Indeed, our results imply that the structural properties may be similar in all groups (decreased material properties but increased crosssectional area). A tissue that needs more material to perform the same task as a tissue with less material is not optimized for its task. It should be noted that structural properties may be more relevant for some clinical significance questions when it comes to function. While this is true, as noted above, this was not the primary purpose of the study.

The use of dumbbell-stamped specimens implies an assumption of isotropy in our samples. This is a limitation of the biomechanical methods. However, the assumption that tendon is linearly isotropic would only be a significant limitation if the goal of our study was to model tissue behavior. The goal of this study was to make relative comparisons between various groups subjected to different injury factors. Therefore, while our simplification may detract from determination of the true tissue properties, it does not prevent us from testing our hypothesis, which required a comparison between single and multiple injury factors. Additionally, all specimens in all groups were treated identically, so comparisons between groups remained valid.

The results of this study have important clinical implications. The findings can help explain some of the variability seen in the clinical setting. It is known that most individuals who perform repetitive overhead activities (overuse) do not develop supraspinatus tendinosis, although a small number do. It is, therefore, likely that other factors contribute to determining who will and who will not develop cuff disease from repetitive activity. These factors could include acute overload injury, increased joint laxity, or limited coracoclavicular arch space. Additionally, there are anatomical variations in the coracoacromial arch (e.g., hooked acromion, reduced area of the supraspinatus outlet, and tight, thickened coracoacromial ligament), which have been correlated with an increased prevalence of rotator cuff tears. ${ }^{1,17,23}$ However, not all individuals with these features will develop cuff disease. The development of cuff tendinosis is likely multifactorial. This study helps to confirm the hypothesis that the development of rotator cuff tendinosis involves multiple factors. Based on these findings it might be possible to identify individuals who are predisposed to developing cuff disease from overhead activities through evaluation of their coracoacromial arch. Activity or job task restrictions for those individuals at risk might decrease the incidence of cuff disease.

There are a number of limitations to the current study. The injury was incited within four weeks of the introduction of the factor, as evidenced by decreased properties at this time point. Since the earliest assay time point of our study was 4 weeks, the progression of the injury could not be measured. Additional early time points would help describe the deterioration of mechanical properties over time.

The mechanism of injury cannot be fully explained solely with the histologic and biomechanical assays performed here. Future studies will, therefore, examine the cellular and molecular changes that occur early on, to determine mechanistic explanations for the changes in material properties. Specifically, the role of inflammatory and growth factors will be assessed. Finally, once the molecular pathways for injury are known, pharmacological therapeutics will be used to specifically target the pathways causing the tendinosis condition.

\section{ACKNOWLEDGMENTS}

This study was supported by a grant from the NIH (R01-AR43179). The authors thank D. Wright for mechanical testing assistance, R. Taylor for histology assistance, J. Charvet for administrative assistance, and P. Robinson for technical assistance.

\section{REFERENCES}

${ }^{1}$ Bigliani, L. U., J. B. Ticker, E. L. Flatow, L. J. Soslowsky, and V. C. Mow. Relationship of acromial architecture to rotator cuff disease. Clin. Sports Med. 10:823-838, 1991.

${ }^{2}$ Bureau of Labor Statistics, Occupational Injuries and Illnesses in the United States by Industry, 1988. Bulletin No. 2368, August 1990.

${ }^{3}$ Carpenter, J. E., C. L. Flanagan, S. Thomopoulos, E. H. Yian, and L. J. Soslowsky. The effects of overuse combined with intrinsic or extrinsic alterations in an animal model of rotator cuff tendinosis. Am. J. Sports Med. 26:801-807, 1998.

${ }^{4}$ Carpenter, J. E., S. Thomopoulos, C. L. Flanagan, C. M. DeBano, and L. J. Soslowsky. Rotator cuff defect healing. A biomechanical and histological analysis in an animal model. J. Shoulder Elbow Surg. 7:599-605, 1998.

${ }^{5}$ Codman, E. A., and I. B. Akerson. The pathology associated with rupture of the supraspinatus tendon. Ann. Surg. 93:348359, 1931.

${ }^{6}$ Jobe, F. W., R. S. Kvitne, and C. E. Giangarra. Shoulder pain in the overhand or throwing athlete. The relationship of anterior instability and rotator cuff impingement in [published erratum in Orthop. Rev. 8:1268, 1989] Orthop. Rev. 18:963$975,1989$.

${ }^{7}$ Leadbetter, W. C. Cell-matrix response in tendon injury. Clin. Sports Med. 11:533-578, 1992.

${ }^{8}$ Lohr, J. F., and H. K. Uhthoff. The microvascular pattern of 
the supraspinatus tendon. Clin. Orthop. Relat. Res. 254:3538, 1990.

${ }^{9}$ McMaster, W., and J. Troup. A survey of interfering shoulder pain in United States competitive swimmers. Am. J. Sports Med. 21:67-70, 1993.

${ }^{10}$ Meyer, A. W. The minute anatomy of attrition lesions. $J$. Bone Jt. Surg. 13:341-360, 1931.

${ }^{11}$ Moseley, H. F., and I. Goldie. The arterial pattern of the rotator cuff of the shoulder. J. Bone Jt. Surg. 45B:780-789, 1963.

${ }^{12}$ Neer, C. S. Shoulder Reconstruction. Philadelphia: Saunders, 1994, $624 \mathrm{pp}$

${ }^{13}$ Ozaki, J., S. Fujimoto, Y. Nakagawa, K. Masuhara, and S. Tamai. Tears of the rotator cuff of the shoulder associated with pathological changes in the acromion. J. Bone Jt. Surg. 70A:1224-1230, 1988.

${ }^{14}$ Rathbun, J. B., and I. Macnab. The microvascular pattern of the rotator cuff. J. Bone Jt. Surg. 52B:540-553, 1970.

${ }^{15}$ Renstrom, P., and R. J. Johnston. Overuse injuries in sports: A review. Sports Med. 2:316-333, 1985.

${ }^{16}$ Sommer, H. M. The biomechanical and metabolic effects of a running regime on the Achilles tendon in the rat. Int. Orthop. 11:71-75, 1987.

${ }^{17}$ Soslowsky, L. J., C. H. An, C. M. DeBano, and J. E. Carpenter. The coracoacromial ligament: In situ load and vis- coelastic properties in rotator cuff disease. Clin. Orthop. Relat. Res. 330:40-44, 1996.

${ }^{18}$ Soslowsky, L. J., J. E. Carpenter, C. M. DeBano, I. Banerji, and M. R. Moalli. Development and use of an animal model for investigations on rotator cuff disease. J. Shoulder Elbow Surg. 5:383-392, 1996.

${ }^{19}$ Soslowsky, L. J., S. Thomopoulos, S. Tun, C. L. Flanagan, C. C. Keefer, J. Mastaw, and J. E. Carpenter. Overuse activity injures the supraspinatus tendon in an animal model: Histologic and biomechanical study. J. Shoulder Elbow Surg. 9:79-84, 2000.

${ }^{20}$ Uhthoff, H. K., and K. Sarkar. Classification and definition of tendinopathies. Clin. Sports Med. 10:707-720, 1991.

${ }^{21}$ Uhthoff, H. K., and H. Sano. Pathology of failure of the rotator cuff tendon. Orthop. Clin. North Am. 28:31-41, 1997.

${ }^{22}$ Yeh, J. K., J. F. Aloia, J. M. Tierney, and S. Sprintz. Effect of treadmill exercise on vertebral and tibial bone mineral content and bone mineral density in the aged adult rat: Determined by dual-energy x-ray absorptiometry. Calcif. Tissue Int. 52:234-238, 1993.

${ }^{23}$ Zuckerman, J. D., F. J. Kummer, F. Cuomo, J. Simon, S. Rosenblum, and N. Katz. Influence of coracoacromial arch anatomy on rotator cuff tears. J. Shoulder Elbow Surg. 1:414, 1992. 